\title{
On the Shoulder of Giants and Luigi Padeletti Is One of Them
}

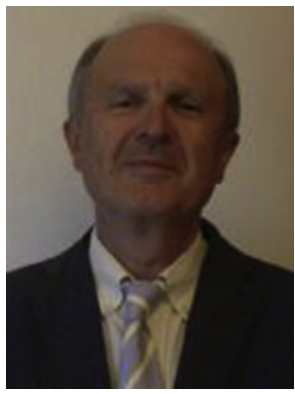

Giuseppe Bagliani, MD

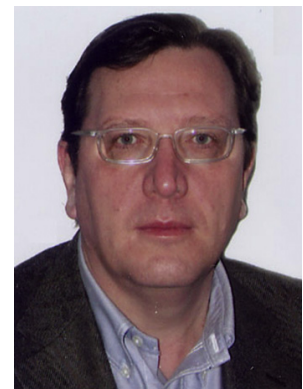

Roberto De Ponti, MD, FHRS

Editors
When, after completing the first issue inspired by the work of the big names in the field of cardiac arrhythmias, we moved on to the second issue, we were so satisfied with the work that we felt as we were "on the shoulders of giants."

The first issue was focused on basic electrocardiography in normal and diseased heart, apparently a very simple topic, but very frequently neglected by the hypertechnologic young generations. Along with Luigi Padeletti, it was decided that a second issue was necessary to complete the body of knowledge on electrocardiographic interpretation in bradycardias, complex tachycardias, and peculiar clinical conditions. This was to remind the readership of the information necessary to use the surface electrocardiogram as a fundamental instrument to orient diagnosis in the universe of cardiac arrhythmias. To this aim, we invited Roberto De Ponti, a friend for ages, to join the editors.

At that time, we would have never expected that and friend Luigi, would have left us by the time the second issue was completed. He passed away on a sad December evening and, as in a nightmare, we are still unable to cope with this. Although he is no longer with us in our professional and personal life, his culture, experience, equilibrium, and savoir faire remain.

Looking at the hard work done and the long years of fruitful cooperation with Luigi, we realize that we have been so lucky to have known a giant like him and to have been on his shoulders. The strength of his example spurs us on to continue the study of and the rational approach to cardiac arrhythmias, both based on the surface electrocardiogram. the person who inspired these issues, our mentor

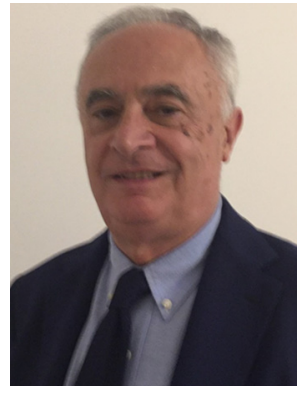

Luigi Padeletti, MD
On behalf of all the contributors, who have been willing to participate in this venture and support the hard work with their scientific and practical experience, we dedicate this issue to Luigi.

Thank you, Luigi. We will never forget you.

$$
\begin{array}{r}
\text { Giuseppe Bagliani, MD } \\
\text { Arrhythmology Unit } \\
\text { Cardiology Department } \\
\text { Foligno General Hospital } \\
\text { Via Massimo Arcamone } \\
\text { Foligno, Perugia 06034, Italy } \\
\text { Cardiovascular Disease Department } \\
\text { University of Perugia } \\
\text { Piazza Menghini 1 } \\
\text { Perugia 06129, Italy } \\
\text { Roberto De Ponti, MD, FHRS } \\
\text { Department of Cardiology } \\
\text { School of Medicine } \\
\text { University of Insubria } \\
\text { Viale Borri, 57 } \\
\text { Varese 21100, Italy } \\
\text { Luigi Padeletti, MD } \\
\text { Heart and Vessels } \\
\text { University of Florence } \\
\text { viale Morgagni, 85 } \\
\text { Florence, Italy } \\
\text { E-mail addresses: }
\end{array}
$$

